\title{
Practical Approach to Hair Loss Diagnosis
}

\author{
Sukhbir Singh ${ }^{1}$ Kumaresan Muthuvel ${ }^{2}$ \\ ${ }^{1}$ Division of General Surgery, Department of Plastic Surgery, \\ Resplendent the Cosmetic Studio, New Delhi, India \\ 2 Department of Dermatology, Cutis Skin Clinic and Hair Transplant \\ Centre, Coimbatore, India
}

\begin{abstract}
Address for correspondence Sukhbir Singh, MS, DNB, Department of Plastic and Cosmetic Surgery, Resplendent the Cosmetic Studio, R-9, Greater Kailash Part 1, New Delhi-110048, India (e-mail: sukhi_1@yahoo.com).
\end{abstract}

Indian J Plast Surg 2021;54:399-403.

\author{
Abstract \\ Keywords \\ - hair loss \\ - Cicatrical alopecia \\ - investigations in hair \\ loss \\ - Tug test
}

Hair loss is one of the common complaints encountered in a cosmetic practice. Evaluating a patient with hair loss requires a thorough understanding of the various causes of hair loss and practical knowledge in hair cosmetics and hair styling procedures. A systematic approach in hair loss evaluation helps the treating physician in arriving at a diagnosis. Various bedside tests and advent of trichoscopy has empowered the physician in arriving at a diagnosis. This article aims to provide the physician a systematic approach in evaluating a patient with hair loss.

\section{Introduction}

Hair loss (alopecia) is one of the common problems encountered among men and women in clinical practice, which is often a major source of distress. There have been various causes documented for alopecia, including scarring alopecia, a nonscarring alopecia, patterned hair loss, telogen effluvium (TE), drug-induced hair loss, hair shaft disorders, and congenital. Hence, a systematic approach has to be taken by the physician, including history taking, clinical examination, laboratory evaluation, and specialized tests in diagnosing the cause of alopecia.

\section{History Taking}

Progressive thinning and excess hair shedding are the two most common complaints from the patients reporting hair loss. In cases of diffuse thinning, hair loss is not noticed until $50 \%$ of scalp hairs are lost. ${ }^{1}$ On an average, shedding of up to 100 telogen hairs per day is considered normal. ${ }^{1}$ A proper and detailed history is important in diagnosing the etiology (-Table 1). Progressive thinning of hair is observed in patterned hair loss (PHL), scarring alopecia, and other dermatological conditions such as psoriasis and seborrheic dermatitis. Acute excessive shedding is seen in TE, anagen effluvium (AE), and during the active stages of scarring alopecia. The age of onset of hair loss provides a clue to the diagnosis; in children, the common causes of hair loss encountered in practice are alopecia areata (AA) and tinea capitis (TC). ${ }^{2}$ Trichotillomania (TTM) is observed in the prepubertal to young adulthood age. ${ }^{2}$ Traction alopecia is usually observed in young adult women. ${ }^{2}$ PHL usually has a postpubertal age of onset. ${ }^{2}$ Frontal fibrosing alopecia (FFA) is usually encountered in postmenopausal women. ${ }^{2}$

The duration of hair loss aids in diagnosis. Conditions such as acute TE, which occurs in response to a variety of triggers, usually lasts for $<6$ months. Acute hair loss is observed in AA and AE. Chronic hair loss is seen in PHL, scarring alopecia, and hair shaft disorders.

Hair loss may be due to breakage or loss from the roots. Hair breakage is observed in TC, hair shaft disorders, TTM, and improper hair care practices. Loss from the roots is observed in TE, AE, and PHL. Scalp itching and soreness are associated with TC, scalp psoriasis, seborrheic dermatitis, and cicatricial alopecia (CA). Trichodynia is usually reported in PHL. ${ }^{3}$ History of any medical or surgical treatment for a period of 6 months prior to the onset of hair loss might help in associating the cause of hair loss. Hair loss is also published online

December 27, 2021
DOI https://doi.org/ 10.1055/s-0041-1739240. ISSN $0970-0358$.

\footnotetext{
(c) 2021. Association of Plastic Surgeons of India. All rights reserved. This is an open access article published by Thieme under the terms of the Creative Commons Attribution-NonDerivative-NonCommercial-License, permitting copying and reproduction so long as the original work is given appropriate credit. Contents may not be used for commercial purposes, or adapted, remixed, transformed or built upon. (https://creativecommons.org/ licenses/by-nc-nd/4.0/)

Thieme Medical and Scientific Publishers Pvt. Ltd., A-12, 2nd Floor, Sector 2, Noida-201301 UP, India
} 
Table 1 History taking checklist

\begin{tabular}{|l|}
\hline Duration of hair loss-acute/chronic \\
\hline Thinning of hair/excess shedding of hair \\
\hline Pattern of hair loss-diffuse/localized \\
\hline Associated symptoms-burning, itching, scaling \\
\hline Past medical and surgical history \\
\hline Drug history \\
\hline Nutritional history \\
\hline Psychosocial history \\
\hline Hair grooming practices \\
\hline Family history of similar hair loss \\
\hline
\end{tabular}

encountered in various auto immune, metabolic, and endocrine disorders. $^{2}$

Drug-induced hair loss can be encountered after a particular period of starting the medications, ranging from days to months. Drug-induced hair loss is diagnosed by excluding other etiologies and correlating the exposure to drugs and the onset of hair loss.. Drug-induced alopecia is usually diffuse and nonscarring. TE, AE, and accentuation of PHL by androgens are encountered secondary to drugs. ${ }^{4}$ The patient's nutritional history is essential in analyzing the etiology of hair loss. Hair loss is observed in nutritional deficiencies and various eating disorders like bulimia and anorexia nervosa and the pattern of hair loss seen is mostly TE. $^{2}$ A detailed psychosocial history aids in diagnosing TTM, $\mathrm{AA}$, and stress-induced TE. ${ }^{5}$ History of hair grooming practices is essential in all cases of hair loss. Hair shaft damage due to a variety of physical and chemical factors leads to hair loss as a result of breakage. TA, hair shaft breakage, and central cicatricial alopecia (CCA) are associated with improper hair care practices. A detailed family history of similar hair loss helps in diagnosing PHL, AA, and congenital hair loss disorders.

In women, history taking should evaluate the menstrual cycles, pregnancies, menopausal symptoms, and the usage of hormonal replacement therapies. History suggestive of polycystic ovarian disease (PCOS) and hypothyroidism should be elicited.

\section{Clinical Examination}

Careful clinical examination (checklist in -Table 2) and algorithmic evaluation of hair loss aids in arriving at the diagnosis (-Fig. 1).

Patient positioning for the examination: Patient should be seated on a chair with good lighting. Hair extensions, hairpieces, and clips if any should be removed before the examination. The scalp should be observed from the top, sides, and occipital region. The density of the hair is compared visually over the different regions of the scalp. A visible scalp indicates $50 \%$ hair loss. ${ }^{1}$

The pattern of hair loss aids in the diagnosis. Patchy hair loss is usually seen in AA and CA. Diffuse hair loss is seen in
Table 2 Clinical examination checklist

\begin{tabular}{|l|}
\hline Patient positioning \\
\hline Overall scalp examination- \\
- Pattern of hair loss \\
- Overall density \\
- Frontal hair line \\
- Other body surface hairs \\
\hline Close-up examination of scalp \\
- Erythema \\
- Hyper-/hypopigmentation \\
- Scaling \\
- Crusting \\
\hline Pair pull test \\
\hline Hair card test \\
\hline Hair tug test \\
\hline Trichoscopy \\
\hline Scalp biopsy \\
\hline
\end{tabular}

TE, AE, and diffuse AA. Frontal hair line recession and vertex hair loss is seen in PHL. More than one pattern of hair loss might coexist. The color and length of the hair should be examined. Eyebrows, eyelashes, and facial and body hair should also be examined. The scalp should be examined for the presence of scaling, erythema, follicular ostia, pigmentation, crusting, and pustules to differentiate scarring from nonscarring alopecia. ${ }^{6}$

Bedside tests to be performed in evaluating hair loss:

1. Card test: The card test is done to differentiate the newly growing hairs from the broken hairs. An $8 \times 12 \mathrm{~cm}$ card, with white and black color on one side to give contrast to the dark and light hair, is used for this test. ${ }^{1}$ The card is placed on the scalp against the hair shafts on the affected area (-Fig. 2). Newly growing hairs are tapered at the ends, broken hairs have blunt ends, and miniaturized hairs have a small caliber than the other hairs. ${ }^{7}$

2. Hair pull test: This is a simple bedside test to assess the presence of active hair loss. ${ }^{1}$ A group of approximately 50 to 60 hairs is grasped between the thumb, index and the middle finger and a gentle pull along the hair shaft from the scalp toward the hair ends should be executed as shown in -Fig. 3. The presence of $>5$ to 6 easily extractable hairs indicates a positive pull test and active hair loss. This test should be done in all the regions of the scalp. Patients should avoid head wash at least 24 hours before the test.

3. Tug test: A tug test is performed to assess the hair fragility. ${ }^{1}$ A group of hairs is held between the hairs in the middle of the shaft length, and with the other hand a pulling force should be executed from the end of the hair shaft, as shown in - Fig. 4. Breakage of hair shaft during the test indicates fragile hair and hair shaft abnormalities.

4. Hair mount: Microscopic hair examination differentiates the disorders of the hair cycle and the hair shaft 


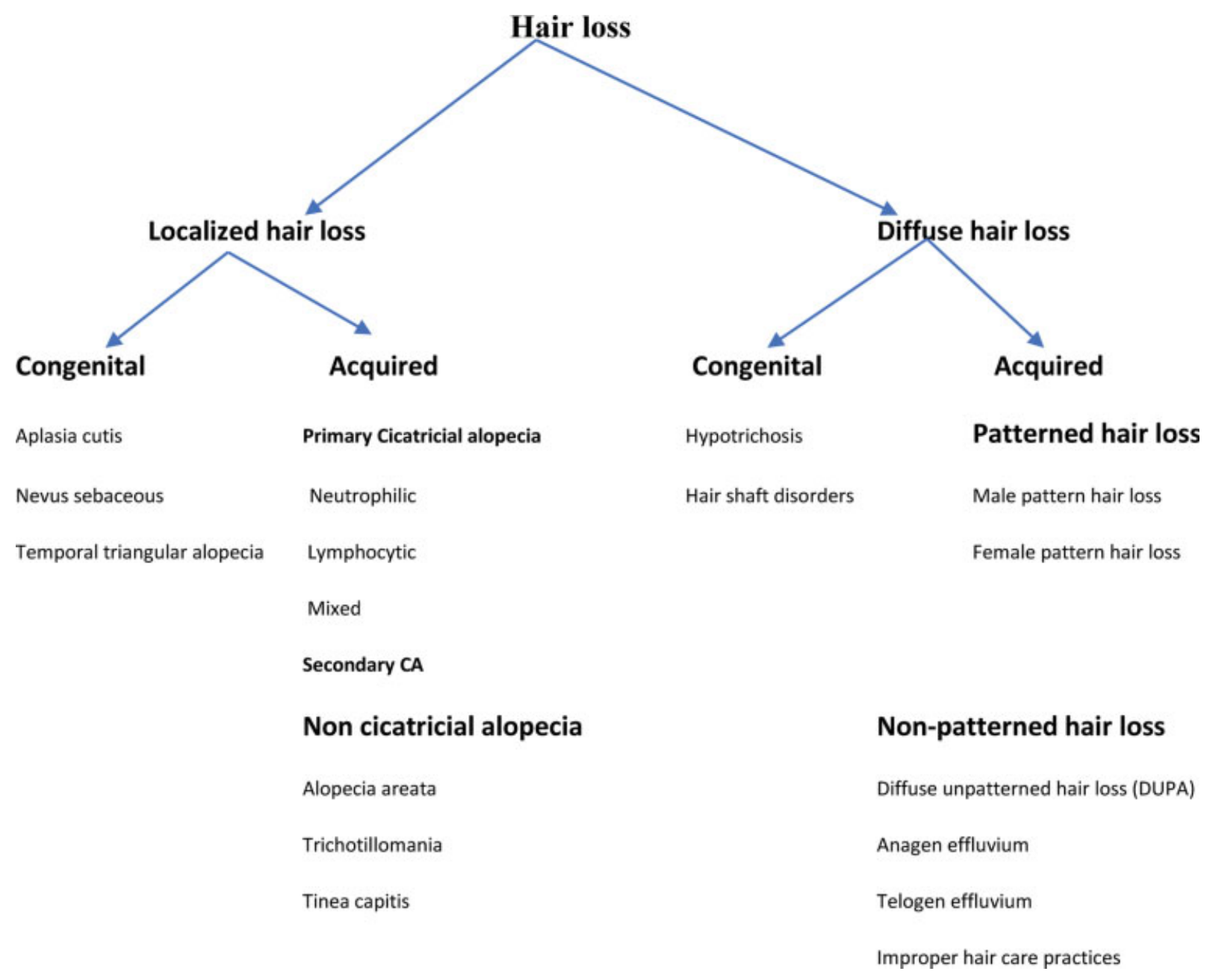

Fig. 1 Algorithm for evaluating hair loss.



Fig. 2 Card test.

abnormalities. ${ }^{1}$ Epilated hairs are mounted on the slides with a mounting medium and a coverslip on the top, and the characters are examined under the light microscope. Trichoscopy has made hair mount test less significant nowadays.

5. Trichoscopy: A simple, quick, noninvasive bedside investigative tool aids in the diagnosis of alopecia ( - Table 3 ).

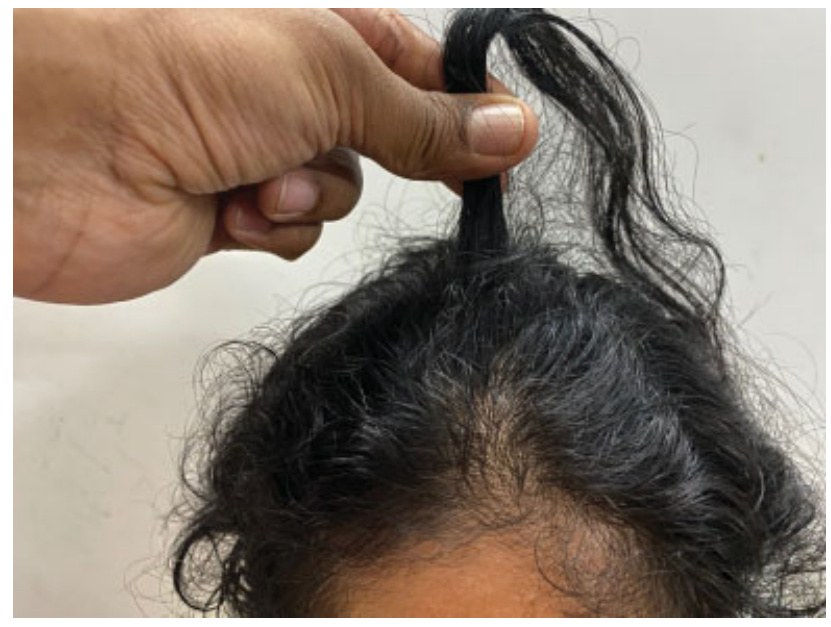

Fig. 3 Hair pull test.

Any dermatoscope can be used as a trichoscope. ${ }^{8}$ Trichoscopes can be used to identify the target area to be biopsied.

6. Scalp biopsy: Scalp biopsy helps to evaluate doubtful cases and unexplained CA. Biopsy specimen should be taken from the active margin of the lesion, with few hair 


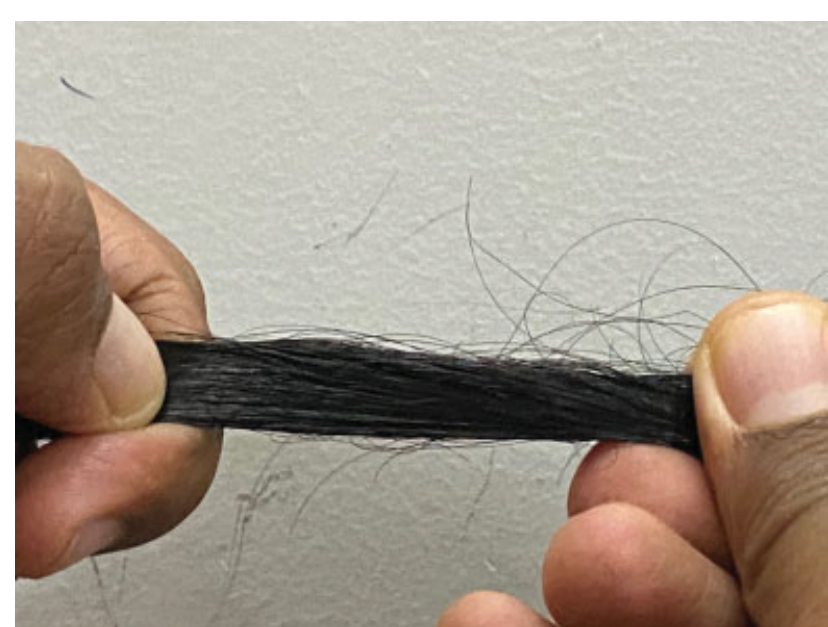

Fig. 4 Tug test.

follicles and a part of subcutaneous fat tissue. It is preferable to harvest two 4-mm biopsy specimens for the horizontal and vertical sectioning. Horizontal sectioning helps to evaluate the follicle at various levels, and vertical sectioning helps to evaluate the entire follicle. ${ }^{11}$

\section{Hair Cosmetic and Hair Loss}

Hair cosmetics has become an integral part of grooming. A knowledge of hair care products, their use, and their possible side effects helps the physician in dealing with hair loss due to hair cosmetics. Hair shaft has a layer of cuticle covered with a special lipid coating, consisting of 18-methyl eicosanoic acid (18-MEA) and free lipids, which impart hydrophobicity to the hair shaft. ${ }^{12}$ Excessive chemical treatment like hair straightening, keratin treatment, improper grooming practices, and environmental exposure damages this lipid coating of cuticle and leads to loss of hydrophobicity of the hair shaft. Hydrophilic hair shaft is prone to mechanical damage due to water absorption and easy breakage due to friction. ${ }^{12}$ The chief ingredients in a shampoo are cleansing agents, additives, conditioning agents, and special care agents. ${ }^{13}$ Aggressive shampooing leads to difficulty in untangling hair and frizzy hair. ${ }^{14}$ Contact dermatitis because of the ingredients in the shampoo is also a major concern. ${ }^{14}$ Grooming dry frizzy hair leads to breakage of hair shaft. The use of conditioners helps in reducing the friction between the hair shafts and makes it easier to groom. ${ }^{12}$ Split ends are formed due to loss of hair cuticle. Hair shaft exposed to the free radicals in the hair cosmetics and sun exposure are prone to splitting and breakage. ${ }^{15}$ Grooming with hair dryer or flat iron should be done with a silicone spreader along the hair shaft to avoid damage to the hair shaft. ${ }^{16}$ Straightening the hair shaft for styling purpose is done by chemical relaxers/heat pressing. Hair fibers are prone to breakage, following hair straightening, due to loss of cysteine bonds, disulfide bonds, hydrogen bonds, and thermal damage. ${ }^{17,18}$ Special cosmetics and grooming practices should be followed following hair straightening to avoid hair breakage and hair loss.

\section{Conclusion}

Evaluating a patient with hair loss requires a systematic approach in history taking, clinical examination, laboratory evaluation, and specialized tests such as trichoscopy/scalp biopsy. A thorough knowledge on the various etiologies and

Table 3 Trichoscopy findings in hair loss

\begin{tabular}{|l|l|}
\hline Condition & Findings \\
\hline Androgenetic alopecia & $\begin{array}{l}\text { Hair shaft thickness heterogeneity (simultaneous presence of thin, intermediate, and thick } \\
\text { hairs), yellow dots, perifollicular discoloration (peripilar sign), an increased proportion of } \\
\text { vellus hairs, and an increased proportion of follicular units with only one emerging hair shaft }\end{array}$ \\
\hline AA & $\begin{array}{l}\text { Yellow dots, black dots, and exclamation } \\
\text { mark hairs. }\end{array}$ \\
\hline TE & $\begin{array}{l}\text { No diagnostic findings. Multiple short upright regrowing hairs may indicate the regrowth } \\
\text { phase of TE. }\end{array}$ \\
\hline Trichotillomania & $\begin{array}{l}\text { Hairs broken at different lengths, short hairs } \\
\text { with trichoptilosis (split ends), coiled hairs, } \\
\text { exclamation mark hairs, and hair shaft residues, flame hairs, the V-sign, hook hairs, hair } \\
\text { powder, and tulip hairs. }\end{array}$ \\
\hline TC & Comma hairs and corkscrew hairs. \\
\hline AE & Black dots, monilethrix like hairs, and exclamation mark hairs \\
\hline Lichen plano pilaris & $\begin{array}{l}\text { Intense perifollicular scaling, elongated linear blood vessels in concentric arrangement and } \\
\text { violaceous inter- or perifollicular violaceous areas. }\end{array}$ \\
\hline Frontal fibrosing alopecia & Lack of follicular openings and minor perifollicular scaling, perifollicular erythema \\
\hline Folliculitis decalvans & Hair tufts that contain 5 to 20 hairs \\
\hline Dissecting cellulitis & Yellow structureless areas and 3-dimensional yellow dots imposed over dystrophic hair shaft. \\
\hline Discoid lupus erythematosus & Large yellow dots, occasionally with superimposed thin blood vessels \\
\hline
\end{tabular}

Abbreviations: AA, alopecia areata; AE, anagen effluvium; TC, tinea capitis; TE, telogen effluvium. 
the clinical manifestations of the various causes of hair loss aids in accurate diagnosis. Accurate diagnosis forms the pillar-stone for prompt treatment in patients of hair loss. This not only leads to positive results but also helps to gain patient confidence and goodwill in the long run.

\section{Financial Disclosure}

None.

\section{Conflict of Interest}

None.

\section{References}

1 Jackson AJ, Price VH. How to diagnose hair loss. Dermatol Clin 2013;31(01):21-28

2 Mubki T, Rudnicka L, Olszewska M, Shapiro J. Evaluation and diagnosis of the hair loss patient: part I. History and clinical examination. J Am Acad Dermatol 2014;(03):415.e1-415.e15

3 Durusoy C, Ozenli Y, Adiguzel A, et al. The role of psychological factors and serum zinc, folate and vitamin B12 levels in the aetiology of trichodynia: a case-control study. Clin Exp Dermatol 2009;34(07):789-792

4 Patel M, Harrison S, Sinclair R. Drugs and hair loss. Dermatol Clin 2013;31(01):67-73

5 Hadshiew IM, Foitzik K, Arck PC, Paus R. Burden of hair loss: stress and the underestimated psychosocial impact of telogen effluvium and androgenetic alopecia. J Invest Dermatol 2004;123(03): 455-457

6 Filbrandt R, Rufaut N, Jones L, Sinclair R. Primary cicatricial alopecia: diagnosis and treatment. CMAJ 2013;185(18):1579-1585
7 Mirmirani P, Huang KP, Price VH. A practical, algorithmic approach to diagnosing hair shaft disorders. Int J Dermatol 2011;50 (01):1-12

8 Mubki T, Rudnicka L, Olszewska M, Shapiro J. Evaluation and diagnosis of the hair loss patient: part II. Trichoscopic and laboratory evaluations. J Am Acad Dermatol 2014;71(03):431. e1-431.e11

9 Ross EK, Vincenzi C, Tosti A. Videodermoscopy in the evaluation of hair and scalp disorders. J Am Acad Dermatol 2006;55(05): 799-806

10 Inui S, Nakajima T, Nakagawa K, Itami S. Clinical significance of dermoscopy in alopecia areata: analysis of 300 cases. Int J Dermatol 2008;47(07):688-693

11 Özcan D, Özen Ö, Seçkin D. Vertical vs. transverse sections of scalp biopsy specimens: a pilot study on the comparison of the diagnostic value of two techniques in alopecia. Clin Exp Dermatol 2011;36(08):855-863

12 Gavazzoni Dias MF. Hair cosmetics: an overview. Int J Trichology 2015;7(01):2-15

13 Deeksha MR, Malviya R, Sharma PK. Advancement in shampoo (a dermal care product): preparation methods, patents and commercial utility. Recent Pat Inflamm Allergy Drug Discov 2014;8 (01):48-58

14 Draelos ZD. Shampoos, conditioners, and camouflage techniques. Dermatol Clin 2013;31(01):173-178

15 Swift JA. The mechanics of fracture of human hair. Int J Cosmet Sci 1999;21(04):227-239

16 Madnani N, Khan K. Hair cosmetics. Indian J Dermatol Venereol Leprol 2013;79(05):654-667

17 Bolduc C, Shapiro J. Hair care products: waving, straightening, conditioning, and coloring. Clin Dermatol 2001;19(04):431-436

18 Shetty VH, Shetty NJ, Nair DG. Chemical hair relaxers have adverse effects a myth or reality. Int J Trichology 2013;5(01):26-28 